\title{
Electronic excitations and the Becke-Johnson potential: The need for and the problem of transforming model potentials to functional derivatives
}

Andreas Karolewski, Rickard Armiento and Stephan Kümmel

\section{Linköping University Post Print}

\section{Tweet}

N.B.: When citing this work, cite the original article.

Original Publication:

Andreas Karolewski, Rickard Armiento and Stephan Kümmel, Electronic excitations and the Becke-Johnson potential: The need for and the problem of transforming model potentials to functional derivatives, 2013, Physical Review A. Atomic, Molecular, and Optical Physics, (88), 5, 052519-1-052519-9.

http://dx.doi.org/10.1103/PhysRevA.88.052519

Copyright: American Physical Society http://www.aps.org/

Postprint available at: Linköping University Electronic Press http://urn.kb.se/resolve?urn=urn:nbn:se:liu:diva-101974 


\title{
Electronic excitations and the Becke-Johnson potential: The need for and the problem of transforming model potentials to functional derivatives
}

\author{
Andreas Karolewski, ${ }^{1}$ Rickard Armiento, ${ }^{2}$ and Stephan Kümmel ${ }^{1, *}$ \\ ${ }^{1}$ Theoretical Physics IV, University of Bayreuth, 95440 Bayreuth, Germany \\ ${ }^{2}$ Department of Physics, Chemistry and Biology (IFM), Linköping University, SE-58183 Linköping, Sweden
}

(Received 20 September 2013; published 25 November 2013)

\begin{abstract}
Constructing approximations for the exchange-correlation (xc) potential in density functional theory instead of the energy appears attractive because it may provide for a way of easily incorporating desirable features such as a particle number discontinuity into xc functionals. However, xc potentials that are constructed directly are problematic: An xc potential that is not a priori derived as a functional derivative of some xc energy functional is most likely not a functional derivative of any density functional at all. This severely limits the usefulness of directly constructed xc potentials, e.g., for calculating electronic excitations. For the explicit example of the Becke-Johnson (BJ) potential we discuss defining corresponding energy expressions by density path integrals. We show that taking the functional derivative of these energies does not lead back to potentials that are close to the $\mathrm{BJ}$ one, and the new potentials do not share the attractive features of the original BJ expression. With further examples we demonstrate that this is a general finding and not specific to the BJ potential form.
\end{abstract}

DOI: 10.1103/PhysRevA.88.052519

PACS number(s): $31.15 . \mathrm{E}-$, 71.15.Mb, 36.40.Vz

\section{INTRODUCTION}

Kohn-Sham density functional theory [1,2] (DFT) and time dependent DFT [3] (TDDFT) are well known for their practical usefulness in a wide range of applications. This is made possible by a variety of exchange-correlation (xc) functional approximations. Typically, the degree of sophistication that is needed for a functional approximation to fulfill its task grows with the degree of complexity of the physics that one aims to describe. A prominent and practically very relevant example of this type are long-range charge-transfer (CT) excitations. In order to be able to calculate them with at least reasonable accuracy, one so far needs to employ computationally demanding highly nonlocal xc functionals as discussed, e.g., in [4-10]. Many of the xc features that are important for describing long-range CT correctly can easily be directly identified in the xc potential. The field-counteracting behavior of the exact Kohn-Sham exchange potential $[11,12]$ and the potential step structure [13] that is related [14] to the integer particle discontinuity [15] are such examples. It has therefore appeared as a promising strategy to switch perspective in xc functional development and develop approximations for the xc potential directly, instead of approximations for the $\mathrm{xc}$ energy.

Specifically, it appeared as a charming perspective to be able to design new $\mathrm{xc}$ potentials as expressions that are semilocal and computationally inexpensive to evaluate, yet able to perform difficult tasks such as the prediction of CT excitations. The Becke-Johnson (BJ) potential [16] is an example of a potential approximation that has shown promise in this regard $[17,18]$. It is defined by the simple expression

$$
v^{\mathrm{BJ}}(\mathbf{r})=v_{\mathrm{x}}^{\mathrm{h}}(\mathbf{r})+v^{\mathrm{c}}(\mathbf{r}),
$$

\footnotetext{
${ }^{*}$ Corresponding author.
}

where $v_{\mathrm{x}}^{\mathrm{h}}(\mathbf{r})$ takes the role of the Coulomb potential of the exchange hole and

$$
v^{\mathrm{c}}(\mathbf{r})=C \sqrt{\frac{2 \tau(\mathbf{r})}{\rho(\mathbf{r})}}
$$

is a correction that can be interpreted as playing the role of the response potential contribution. The noninteracting kinetic energy density $\tau(\mathbf{r})=2 \sum_{i=1}^{N} \frac{1}{2}\left|\nabla \varphi_{i}(\mathbf{r})\right|^{2}$ is evaluated from the $N$ occupied Kohn-Sham orbitals $\varphi_{i}$, and $C=\frac{1}{\pi} \sqrt{\frac{5}{12}}$. Here and in the following we assume a non-spin-polarized system for clarity and use hartree atomic units. It was shown that $v^{\mathrm{BJ}}$ is a good approximation to the exact exchange potential [16-19] including many of its important features such as the step structure, the derivative discontinuity, and a $\sim-\frac{1}{r}$ asymptotic behavior. In Ref. [17] it was demonstrated that the BJ potential shows features that are closely related to the discontinuous potential changes that occur in the exact exchange Kohn-Sham potential due to the derivative discontinuity. However, in the presence of an electric field the expression does not counteract the applied field and therefore, generalizations for this case were developed $[17,20]$. For one of these generalizations it was explicitly verified that it reliably predicts the static polarizabilities of acetylene oligomers [18], a capability closely connected to the accurate description of exact exchange features. The BJ potential was also applied [21] for the calculation of band gaps and improved results were found with a further modified expression [22]. Since the presence of the derivative discontinuity and other properties of the exchange potential are important for the description of ionization processes and CT $[4,23]$ this simple potential expression is an ideal starting point for our purpose.

Most remarkably, though, is the fact that, except for the correct asymptotic behavior, the important properties that model exact exchange in the BJ potential arise from $v^{\mathrm{c}}-\mathrm{a}$ solely semilocal expression. Via the orbital dependent $(\tau)$ term it incorporates the above mentioned features of exact exchange 
that are usually obtained from expressions that are nonlocal with respect to the orbitals. The low computational cost that is implied by semilocality makes the BJ potential very interesting from an application point of view.

However, potentials that are constructed as direct approximations and not obtained as functional derivatives of an energy functional have a significant downside. They are typically not the functional derivative of any density functional. This is in particular true for the BJ potential $[18,24]$. Such potentials do not comply with the requirements of Kohn-Sham theory [2] and from a formal perspective, their use is not justified. One could hope that this might be just a formal argument and that from a pragmatic point of view one may use such potentials and obtain good results. The Kriger-Li-Iafrate potential approximation [25] to the exact exchange optimized effective potential [26-28] is an example of a potential that is not a functional derivative, but nevertheless is very useful in practice.

However, a potential that is not a functional derivative is problematic also from a pragmatic point of view. First and most obviously, if there is no energy corresponding to the potential, then any form of a consistent energy minimizing calculation is impossible. Second, potentials that are not functional derivatives lack properties that proper xc potentials have, such as rotational and translational symmetry [29]. As a consequence they violate the zero-force theorem [30]. Especially in TDDFT this leads to serious problems as the TD Kohn-Sham equations can no longer be solved stably $[31,32]$.

In this article we study ways that allow one to map xc potentials that are not functional derivatives to new potentials that are functional derivatives of some energy expression, with the aim of finding a map that preserves the relevant features of the original potential. This effort is motivated by the BJ potential that we see as an important step in the quest to find an easy-to-evaluate, computationally efficient semilocal functional that incorporates other nonlocal properties, such as a discontinuity at integer particle numbers, and thus, may be able able to predict CT excitations. In the following we therefore focus on this example. However, most of the conclusions and results that we obtain from the analysis and modification of the BJ expression are also valid for other direct potential approximations that are not functional derivatives.

Our article is outlined as follows. In Sec. II we demonstrate that the BJ potential leads to problems when used in TDDFT, e.g., due to the violation of the zero-force theorem. Following this analysis we explain routes to modify $v^{\mathrm{BJ}}$ with the aim to obtain a potential that is a functional derivative. In Sec. III we discuss semilocal approximations for the hole potential of Eq. (1) in order to convert the whole potential into a semilocal expression. Thereafter, we define different energy expressions corresponding to $v^{\mathrm{c}}$ and take their functional derivative to derive new potentials. By comparing these newly defined expressions with $v^{\mathrm{c}}$ we discuss the prospects of this potential transformation approach (Sec. IV) and close with conclusions in Sec. V.

\section{BJ POTENTIAL IN TDDFT}

In this section we demonstrate which type of results are to be expected when the BJ potential is used in TDDFT. We have implemented $v^{\mathrm{BJ}}$ of Eq. (1) in our customized, time dependent version [33] of the PARSEC [34] real-space code. The time dependent Kohn-Sham equations [3] are propagated in real time [35] on a real-space grid. For the xc potential we use the $\mathrm{BJ}$ expression in the adiabatic approximation, i.e.,

$$
v^{\mathrm{a}-\mathrm{BJ}}(\mathbf{r}, t)=\left.v^{\mathrm{BJ}}\left(\left[\left\{\varphi_{i}\right\}\right], \mathbf{r}\right)\right|_{\left\{\varphi_{i}\right\}=\left\{\varphi_{i}(\mathbf{r}, t)\right\}} \cdot
$$

Our main interest in the TDDFT implementation of the BJ potential is the calculation of excitation energies. One might hope that, just as $v^{\mathrm{BJ}}(\mathbf{r})$ is close to the exact exchange potential in ground-state DFT, the adiabatic extension of the BJ potential $v^{\mathrm{a}-\mathrm{BJ}}(\mathbf{r}, t)(\mathrm{a}-\mathrm{BJ})$ in TDDFT may exhibit important features of the time dependent exact exchange potential. As the ground-state BJ potential shows step structures that are related to the derivative discontinuity in DFT [15] and TDDFT [23,36], and as furthermore the a-BJ potential depends on the orbitals at time $t$, which themselves depend on the density at all prior times $t^{\prime}$ [37], the BJ potential in principle contains the elements that are considered necessary for capturing the spatial and temporal nonlocalities that are required for the description of $\mathrm{CT}$ excitations.

In order to obtain excitation energies from the propagation of the time dependent Kohn-Sham equations we apply a small boost $\exp \left(i \mathbf{r} \cdot \mathbf{k}_{\text {boost }}\right)$ to the ground-state Kohn-Sham orbitals and calculate the time dependent dipole moment $[35,38]$

$$
\mathbf{d}(t)=-\int d^{3} r \mathbf{r} \rho(\mathbf{r}, t) .
$$

From the Fourier transform $\tilde{\mathbf{d}}$ of the dipole moment one obtains the dipole power spectrum

$$
D(\omega)=\sum_{i=1}^{3}\left|\tilde{d}_{i}(\omega)\right|^{2}
$$

whose peak positions indicate the excitation energies [33,35]. As test cases we chose Na clusters. Their excitations are ordinary valence excitations that exhibit no CT. They are ideal for the purpose of testing $v_{\mathrm{xc}}$ approximations via propagation because they are known to be very sensitive to functional inconsistencies $[31,39,40]$ while at the same time convergence with respect to numerical parameters such as time step and grid spacing is relatively easy to achieve.

As a first test we calculated the dipole power spectrum of the sodium dimer and compare it to the adiabatic local density approximation (a-LDA) and the adiabatic Krieger-Li-Iafrate approximation [25] of the exact exchange potential (a-xKLI) in Fig. $1 . \mathrm{Na}_{2}$ is one of the systems which allowed for stable propagation in earlier tests [31] of the a-xKLI approximation, and we observe the same for the a-BJ approximation. Furthermore, the result shows that the a-BJ approximation for $\mathrm{Na}_{2}$ leads to excitations that do not coincide with the ones from a-XKLI, but the transitions are at reasonable positions when compared to experimental results.

As a second test we investigated $\mathrm{Na}_{5}$, a system that by now can be considered an established test case: It has been shown in previous studies that using potentials that are not functional derivatives in the propagation of the $\mathrm{Na}_{5}$ orbitals leads to instabilities in the propagation $[31,39,40]$. We observe this effect so pronouncedly with the a-BJ approximation for $\mathrm{Na}_{5}$ that we are not able to calculate an excitation energy spectrum at all. Even worse, when the system is propagated 


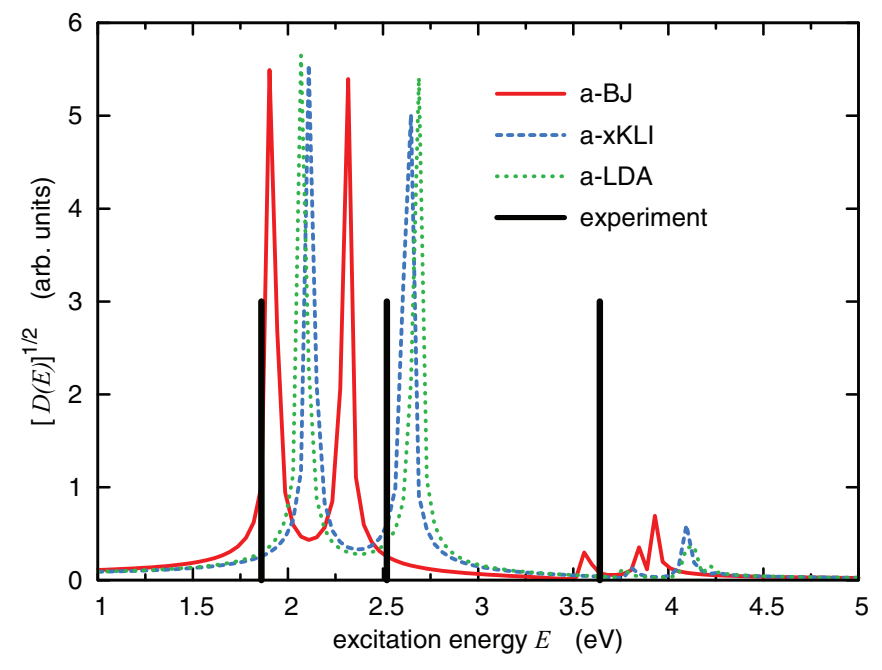

FIG. 1. (Color online) Dipole power spectrum of $\mathrm{Na}_{2}$ for a-BJ, a-xKLI, and a-LDA after a boost energy of $E_{\text {boost }}=10^{-5} \mathrm{eV}$, calculated with a time step of $0.003 \mathrm{fs}$ and a total propagation time of $100 \mathrm{fs}$. Experimental excitation energies from Refs. [41,42] are indicated by vertical black lines (only the indicated energy is relevant, not the length of the line).

with the a-BJ potential without an external TD potential or a boost, i.e., propagated such that the orbitals should only acquire a trivial phase factor, a TD dipole moment of increasing magnitude develops.

We relate this finding to a violation of the zero-force theorem [30]

$$
\int d^{3} r \rho(\mathbf{r}, t) \nabla v_{\mathrm{xc}}(\mathbf{r}, t)=0
$$

for the xc potential $v_{\mathrm{xc}}$. The zero-force condition is not obeyed if a potential expression is used that is not a functional derivative of some energy functional. For $\mathrm{Na}_{5}$ the violation of Eq. (6) is particularly severe. We demonstrate this by showing the left-hand side of Eq. (6) as a function of time in Fig. 2. The zero-force violation grows exponentially in time,

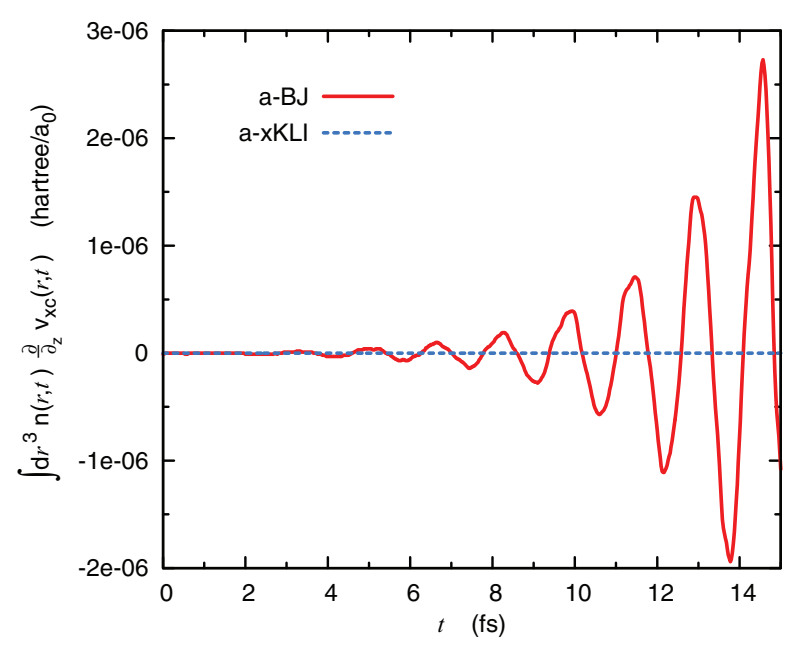

FIG. 2. (Color online) Left-hand side of Eq. (6) (zero-force theorem) for the $z$ component as a function of time for the propagation of the ground state (no boost applied) of $\mathrm{Na}_{5}$. Solid line: a-BJ. Dashed line: a-xKLI. leading to a fast self-excitation of the system. For reasons of comparison Fig. 2 also shows the left-hand side of Eq. (6) evaluated for the a-xKLI potential. Although a-xKLI also does not obey Eq. (6) and leads to a severe violation if a boost is applied [31], Fig. 2 shows that the zero-force violation of a-XKLI is negligible on the scale of the a-BJ violation when no boost is applied. Thus, Fig. 2 illustrates that the problems one has to expect due to the zero-force violation are more severe for a-BJ than for a-xKLI. The comparison also reveals that $v^{\mathrm{c}}(\mathbf{r})$ is the problematic part of a-BJ and the main source of the self-excitation, because the hole potential $v_{\mathrm{x}}^{\mathrm{h}}(\mathbf{r})$ (which is also not a functional derivative) is also part of the a-xKLI potential. Problems with $v^{\mathrm{c}}(\mathbf{r})$ are also expected since the evaluation of this term requires dividing by the density, and as the density becomes very small in the asymptotic regions of any finite system this may lead to numerical instabilities that may intensify the problems that arise from the violation of the zero-force theorem. Although there also is a division by the density in the a-xKLI potential, it is much less problematic there because the density is asymptotically dominated by the highest occupied orbital's density, and the latter appears in the numerator of the a-xKLI potential. Thus, numerical inaccuracies in the denominator can be canceled by the same inaccuracies in the numerator [32].

Finally, the comparison of the two tests, $\mathrm{Na}_{2}$ and $\mathrm{Na}_{5}$, shows that the degree to which the violation of the zero-force condition manifests in practical calculations with the a-BJ potential does depend on the particular system that is studied. This is in line with similar observations for other xc potential approximations $[31,39,40]$. However, in any case our results show that the a-BJ potential as such can hardly be used for reliable TDDFT calculations. Moreover, as the a-BJ potential in its present form cannot be used reliably in TDDFT even for valence excitations (which are typically easier to get right than CT ones), hopes that it could be used for properly describing CT excitations are minimal. We thus did not explore this option any further.

\section{SEMILOCAL REPLACEMENT OF THE SLATER POTENTIAL}

Whereas the TDDFT tests reveal that $v^{\mathrm{c}}(\mathbf{r})$ has problematic aspects, it is also necessary to change $v_{\mathrm{x}}^{\mathrm{h}}(\mathbf{r})$ if one wants to take full advantage of the possibilities that a BJ-like approach offers. In fact, it has already been pointed out in Ref. [16] that the first step for improving the BJ potential would be choosing a semilocal approximation for the hole potential $v_{\mathrm{x}}^{\mathrm{h}}$. There are two motivations for seeking such a replacement. One is the aim to turn the BJ potential into a functional derivative. This topic will be discussed in detail in Sec. IV. The other and even more obvious one is the great increase in computational efficiency that can be achieved by avoiding the many integrations that are needed in the evaluation of the Coulomb potential of the exact exchange hole, i.e., the Slater potential [43]

$$
v_{\mathrm{x}}^{\mathrm{h}}(\mathbf{r})=v_{\mathrm{x}}^{\text {Slater }}(\mathbf{r})=-\int d^{3} r^{\prime} \frac{\hat{\rho}_{\mathrm{x}}\left(\mathbf{r}, \mathbf{r}^{\prime}\right)}{\left|\mathbf{r}-\mathbf{r}^{\prime}\right|},
$$

where the exchange hole is

$$
\hat{\rho}_{\mathrm{X}}\left(\mathbf{r}, \mathbf{r}^{\prime}\right)=\frac{2\left|\sum_{i=1}^{N} \varphi_{i}^{*}(\mathbf{r}) \varphi_{i}\left(\mathbf{r}^{\prime}\right)\right|^{2}}{\rho(\mathbf{r})} .
$$

So far, Eq. (7) was used in the BJ potential. 
In the following we compare the exchange hole potential of different semilocal exchange functionals to the Slater potential and investigate whether any of these approximations can serve as a replacement. We obtain the hole potentials by appropriately factorizing the energy according to [44]

$$
E_{\mathrm{x}}=\int \frac{1}{2} \rho(\mathbf{r}) v_{\mathrm{x}}^{\mathrm{h}}(\mathbf{r}) d^{3} r
$$

with $E_{\mathrm{x}}$ being the exchange energy. A summary of all hole potentials can be found in Appendix A.

In Fig. 3(a) we analyze different hole potentials for the $\mathrm{Be}$ atom as a function of the radial coordinate $r$. We compare the Slater potential with the exchange hole potentials of the Becke-Roussel (BR) [44], the Becke 88 (B88) [45], the Perdew-Burke-Ernzerhof (PBE) [46], the Perdew-Wang 86 (PW86) [47], the Becke 86 (B86) [48], and the local density approximation (LDA) exchange potential expressions. In Fig. 3(b) we show the inverse of these potential expressions for the same atom making the analysis of the asymptotic behavior more convenient. The BR hole potential is the one closest to the Slater potential, especially with respect to the $\sim-\frac{1}{r}$ asymptotic decay. The second best approximation is the B88 hole potential which also has the correct $\sim-\frac{1}{r}$ asymptotic behavior $[45,49]$. The LDA, B86, and PBE curves decay exponentially and as a consequence a modified $v^{\mathrm{BJ}}$ using one of these for the hole potential would lead to a qualitatively wrong asymptotic behavior. Figure 3 also shows that none of the plotted hole potentials approximates the Slater potential well in the center of the system. Here, the BR hole potential is even further off than the other approximations.

In Fig. 4 we performed the same analysis for a more extended system, the oligoacetylene $\mathrm{C}_{6} \mathrm{H}_{8}$ and plotted the corresponding exchange hole potentials along the direction of the molecular backbone (the plotting axis, the $x$ axis, is centered between the $\mathrm{C}$ atoms). Qualitatively the observations here are the same as for the Be atom. In the center of the molecule all potentials have a similar structure. However, for all potentials except for the BR these structures are not as pronounced as for the Slater potential. B86, PW86, PBE, and B88 are very close to each other in the interior region of the molecule.

Among the available semilocal expressions the BR potential thus appears as the closest approximation to the Slater potential, followed by the B88 potential. Indeed, with some success previous studies $[16,21,22]$ have already used the BR [44] instead of the Slater potential in the BJ approach. However, as explained in the next section, for our purposes of testing whether a potential can be constructed that is close to the $\mathrm{BJ}$ model yet at the same time a functional derivative, it is helpful to know the potential explicitly in terms of the density. This rules out the BR approximation and we therefore choose B88 for the following study, as it can be written explicitly in terms of the density while it still provides a reasonable approximation to the Slater potential.

\section{CONSTRUCTION OF A FUNCTIONAL DERIVATIVE}

In this section we will explore ways of how a modified $\mathrm{BJ}$ expression that depends on the density semilocally can be turned into a potential that is a functional derivative of an
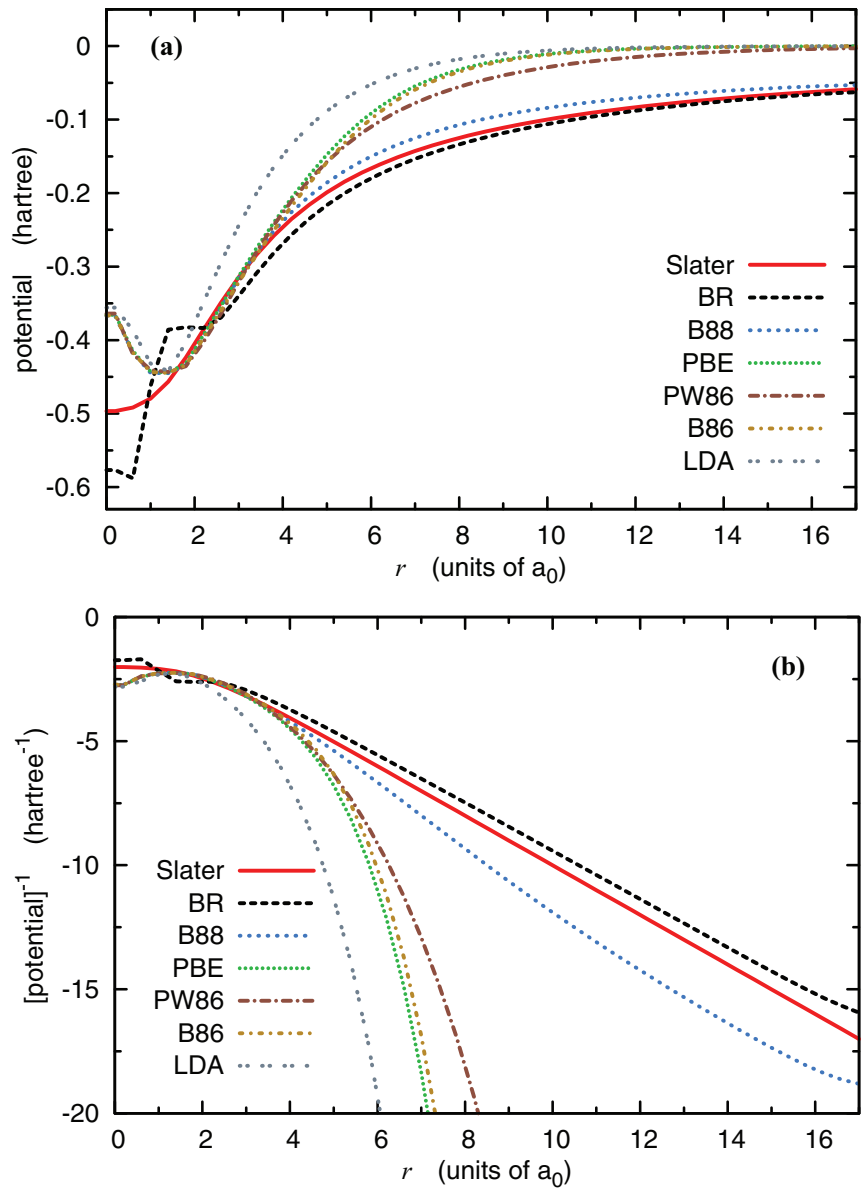

FIG. 3. (Color online) Comparison of different Coulomb hole potentials for the Be atom. See main text for details.

energy functional. To this end we use a line integral [50] in density space to define an energy functional for a potential $v$ that is not a functional derivative

$$
E^{\mathrm{new}}[\rho]=\int_{0}^{1} d \lambda \int d^{3} r v\left(\left[\rho_{\lambda}\right], \mathbf{r}\right) \frac{d \rho_{\lambda}(\mathbf{r})}{d \lambda}
$$

with the density path $\rho_{\lambda}(\mathbf{r})$ and $\rho_{\lambda=0}(\mathbf{r})=0$ and $\rho_{\lambda=1}(\mathbf{r})=$ $\rho(\mathbf{r})$. Defining a new energy functional for a potential that is not a functional derivative by using Eq. (10) was explored in Ref. [18] for a special line integral, the Levy-Perdew virial relation [30], and also for the exchange potential approximation of van Leeuwen and Baerends [51] for two different density paths in Ref. [52].

Once one has defined an energy via Eq. (10) one can calculate the potential that corresponds to this energy by taking the functional derivative with respect to the density,

$$
\tilde{v}=\frac{\delta E^{\mathrm{new}}[\rho]}{\delta \rho} .
$$

In the following we investigate this procedure for the correction term $v^{\mathrm{c}}(\mathbf{r})=C \sqrt{\frac{2 \tau(\mathbf{r})}{\rho(\mathbf{r})}}$ of Eq. (2). The decisive question is whether the newly defined potential $\tilde{v}^{\mathrm{c}}$, which is a proper functional derivative of the energy functional $E^{\text {new }}$, is still close to the original potential $v^{\mathrm{c}}$. Note that if one would insert a potential into Eq. (10) that already is a functional derivative of an energy functional, then the line integral would restore 

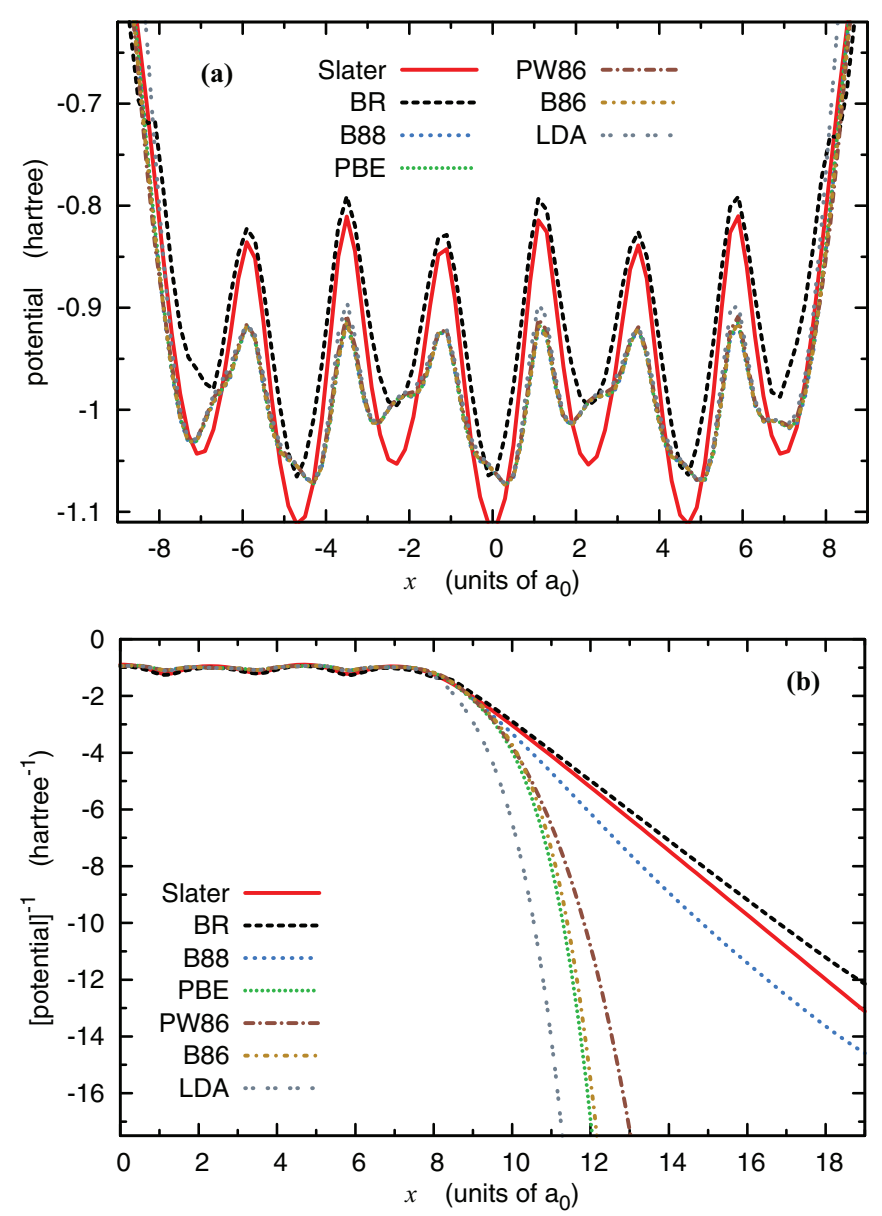

FIG. 4. (Color online) (a) Coulomb hole potential approximations and (b) the inverse Coulomb hole potential approximations of the $\mathrm{C}_{6} \mathrm{H}_{8}$ molecule.

exactly this energy functional and consequently Eq. (11) would give back the potential that was inserted.

For the general BJ expression there is a further technical hurdle. Due to the orbital dependence of $v^{\mathrm{c}}$ via $\tau$ we would have to evaluate the functional derivative of Eq. (11) in an optimized effective potential approach [26-28] and not as a direct analytical derivative. To avoid this difficulty we restrict our investigation to one-orbital systems with densities $\rho_{1}(\mathbf{r})=$ $\left|\varphi_{1}(\mathbf{r})\right|^{2}$. In this case the difference between the functional derivative with respect to the density and the one with respect to the orbital becomes trivial,

$$
\frac{\delta E}{\delta \rho_{1}(\mathbf{r})}=\frac{1}{\varphi_{1}^{*}(\mathbf{r})} \frac{\delta E}{\delta \varphi_{1}(\mathbf{r})},
$$

making superfluous the optimized effective potential procedure: $\tilde{v}^{\mathrm{c}}=u^{\mathrm{c}}=u_{1}^{\mathrm{c}}=\frac{1}{\varphi_{1}^{*}} \frac{\delta E^{\mathrm{new}}}{\delta \varphi_{1}}$ can readily be calculated and compared to $v^{\mathrm{c}}$.

First, we explore this procedure with the straight path (SP)

$$
\rho_{\lambda}(\mathbf{r})=\lambda \rho(\mathbf{r})
$$

When we use the orbital scaling $\varphi_{i, \lambda}(\mathbf{r})=\sqrt{\lambda} \varphi_{i}(\mathbf{r})$ with $v^{\mathrm{c}}$ we obtain the energy integral [from Eq. (10)]

$$
E^{\mathrm{SP}, \mathrm{c}}=\int d^{3} r C \sqrt{2 \tau(\mathbf{r}) \rho(\mathbf{r})} .
$$

From this we obtain the orbital specific potential

$$
\begin{aligned}
u_{i}^{\mathrm{SP}, \mathrm{c}} & =\frac{1}{\varphi_{i}^{*}} \frac{\delta E^{\mathrm{SP}, \mathrm{c}}}{\delta \varphi_{i}(\mathbf{r})} \\
& =C\left\{\sqrt{\frac{\tau(\mathbf{r})}{2 \rho(\mathbf{r})}}-\frac{1}{(2)^{3 / 2}} \nabla\left(\frac{\sqrt{\rho(\mathbf{r})} \nabla \varphi_{i}^{*}(\mathbf{r})}{\sqrt{\tau(\mathbf{r})}}\right) \frac{1}{\varphi_{i}^{*}(\mathbf{r})}\right\} .
\end{aligned}
$$

Second, we use the uniform scaling path (USP)

$$
\rho_{\lambda}(\mathbf{r})=\lambda^{3} \rho(\lambda \mathbf{r}) .
$$

The orbital scaling for the uniform scaling path is $\varphi_{i, \lambda}(\mathbf{r})=$ $\lambda^{3 / 2} \varphi_{i}(\lambda \mathbf{r})$. Since $v^{\mathrm{c}}$ fulfills the scaling relation of the exchange potential

$$
v^{\mathrm{c}}\left(\left[\rho_{\lambda},\left\{\varphi_{i, \lambda}\right\}\right], \mathbf{r}\right)=\lambda v^{\mathrm{c}}\left(\left[\rho,\left\{\varphi_{i}\right\}\right], \lambda \mathbf{r}\right)
$$

we obtain for the line integral [Eq. (10)]

$$
E^{\mathrm{USP}, \mathrm{c}}=\int d^{3} r v^{\mathrm{c}}(\mathbf{r})[3 \rho(\mathbf{r})+\mathbf{r} \cdot \nabla \rho(\mathbf{r})] .
$$

This is the Levy-Perdew exchange virial relation [30]. The orbital functional derivative of this integral is

$$
\begin{aligned}
u_{i}^{\mathrm{USP}, \mathrm{c}}= & \frac{1}{\varphi_{i}^{*}} \frac{\delta E^{\mathrm{USP}, \mathrm{c}}}{\delta \varphi_{i}(\mathbf{r})} \\
= & -C\left\{[3 \rho+(\mathbf{r} \cdot \nabla) \rho] \sqrt{\frac{\tau}{2 \rho^{3}}}+(\mathbf{r} \cdot \nabla) \sqrt{\frac{2 \tau}{\rho}}\right. \\
& +\nabla\left[[3 \rho+(\mathbf{r} \cdot \nabla) \rho] \sqrt{\frac{1}{2 \tau \rho}}\right] \frac{\nabla \varphi_{i}^{*}}{\varphi_{i}} \\
& \left.+[3 \rho+(\mathbf{r} \cdot \nabla) \rho] \sqrt{\frac{1}{2 \tau \rho}} \frac{\nabla^{2} \varphi_{i}^{*}}{\varphi_{i}^{*}}\right\} .
\end{aligned}
$$

In Fig. 5 we compare $u^{\mathrm{SP}, \mathrm{c}}$ and $u^{\mathrm{USP}, \mathrm{c}}$ to $v^{\mathrm{c}}$ for two different one-orbital densities. For part (a) we used an exponential function as the orbital and for part (b) a Gaussian function. Both graphs clearly demonstrate that $u^{\mathrm{SP}, \mathrm{c}}$ and $u^{\mathrm{USP}, \mathrm{c}}$ strongly differ from $v^{\mathrm{c}}$. The qualitative difference is particularly striking in the case of the exponential function where $v^{\mathrm{c}}$ is constant (and supposed to be so for physical reasons [16]), whereas the newly derived potentials vary considerably. We also performed the test for other one-orbital densities, e.g., the $2 p$ or $3 s$ hydrogen orbitals, and obtained deviations of at least similar degree.

One could hope that the finding that the line-integral transformation changes the form of $v^{\mathrm{c}}$ substantially is unproblematic because ultimately one is interested in the transformation of the sum $v_{\mathrm{x}}^{\mathrm{h}}+v^{\mathrm{c}}$. Hence, it is a possibility that the undesired features introduced by the line-integral transformation of $v^{\mathrm{c}}$ are compensated by opposite features introduced in the lineintegral transformation of $v_{\mathrm{x}}^{\mathrm{h}}$. We investigate this possibility for the practically relevant case of the B88 hole potential. In Appendix $\mathrm{B}$ we show the transformation procedure for the B88 hole potential $\left[v_{\mathrm{x}}^{\mathrm{h}, \mathrm{B} 88}\right.$, Eq. (A4)] and calculate the functional derivative of the energy defined by Eq. (10) for the uniform scaling path. In this case it is possible to take the functional derivative with respect to the density directly. Figure 6 compares the newly defined potential $v_{\mathrm{x}}^{\mathrm{USP}, \mathrm{h}, \mathrm{B} 88}$ with the original B88 hole potential $v_{\mathrm{x}}^{\mathrm{h}, \mathrm{B} 88}$ for the exponential and 

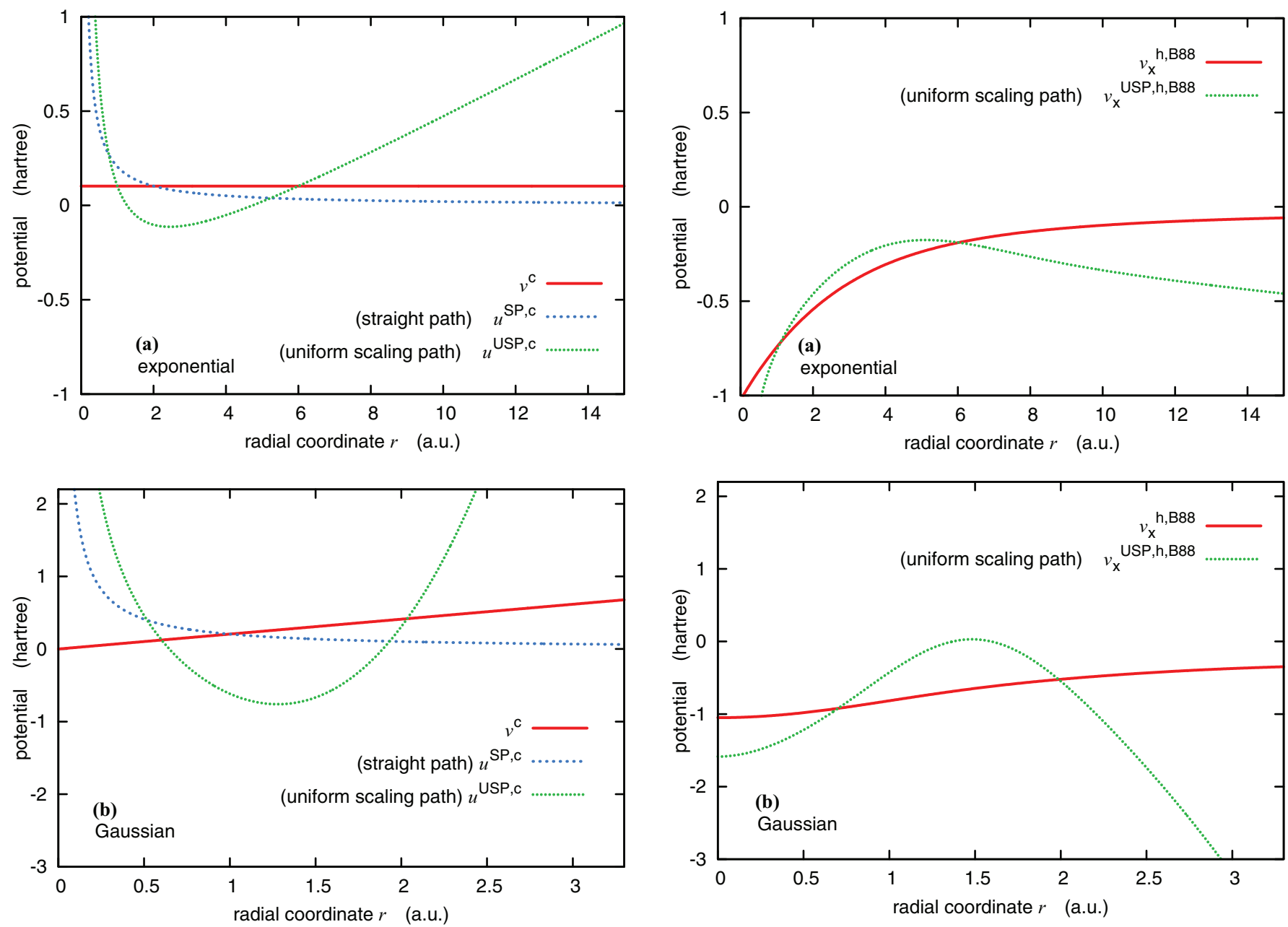

FIG. 5. (Color online) The potential expressions $v^{\text {c }}$ [Eq. (2)], $u^{\mathrm{SP}, \mathrm{c}}$ [Eq. (15)], and $u^{\mathrm{USP}, \mathrm{c}}[\mathrm{Eq}$. (19)] for the exponential (a) and the Gaussian (b) spherical one-orbital densities.

Gaussian one-orbital densities. Similar to the transformation of $v^{\mathrm{c}}$ we observe strong deviations from the original hole expression. The most important features of the hole potential in the $\mathrm{BJ}$ expression - providing the overall potential structure and in particular the correct asymptotic behavior-are lost. We further see that indeed undesired features in the two transformed potentials can (at least in principle) cancel, because the deviations of $v_{\mathrm{x}}^{\mathrm{USP}, \mathrm{h}, \mathrm{B} 88}$ from $v_{\mathrm{x}}^{\mathrm{h}, \mathrm{B} 88}$ are of opposite sign as the deviations of $u^{\mathrm{USP}, \mathrm{c}}$ from $v^{\mathrm{c}}$. To check the extent of the cancellation Fig. 7 shows the sums $v_{\mathrm{x}}^{\mathrm{USP}, \mathrm{h}, \mathrm{B} 88}+v^{\mathrm{USP}, \mathrm{c}}$ and $v_{\mathrm{x}}^{\mathrm{h}, \mathrm{B} 88}+v^{\mathrm{c}}$. For intermediate values of $r$ there is a certain cancellation, but for small and large $r$ the discrepancies remain large.

We therefore conclude that the uniform density scaling and the straight path energy expressions define energy functionals whose functional derivatives are very different from the original $v^{\mathrm{BJ}}$, despite two prior observations that one may have interpreted as suggesting otherwise: First, the form of $v^{\mathrm{BJ}}$ appears to be reasonable close to the exact exchange potential [16-18] and secondly, $E^{\text {USP }}$ yields energy values close to exact exchange values [19]. Our results are consistent with Ref. [52] where the transformation of the Leeuwen-Baerends exchange potential [51] along the same density paths that we used here

FIG. 6. (Color online) The potential expressions $v_{\mathrm{x}}^{\mathrm{h}, \mathrm{B} 88}$ [Eq. (A4)] and $v_{\mathrm{x}}^{\mathrm{USP}, \mathrm{h}, \mathrm{B} 88}$ [Eq. (B2)] for the exponential (a) and the Gaussian (b) spherical one-orbital densities.

also lead to considerable deviations from the original potential for the $\mathrm{Kr}$ atom.

\section{CONCLUSION}

The $\mathrm{BJ}$ potential is not a functional derivative and therefore violates the zero-force theorem. In Sec. II we demonstrated that the theorem is not only violated in principle but also in practice and on a very relevant scale. Thus, the BJ potential as such is not applicable as a cost effective semilocal functional for the calculation of excitations.

After choosing the B88 hole potential as an appropriate semilocal replacement for the Slater potential we analyzed a procedure for transforming potentials which are not functional derivatives into ones that are. The procedure uses a line integral of a given potential expression along a certain density path to define a new energy functional. We investigated two density paths for transforming $v^{\mathrm{c}}$. Comparing the newly defined potentials with the original expression for the case of one-orbital densities we found that the new potentials differ substantially from the original expression. The one-orbital test is a very relevant test because the density far away from a finite system's center is always dominated by one orbital that 

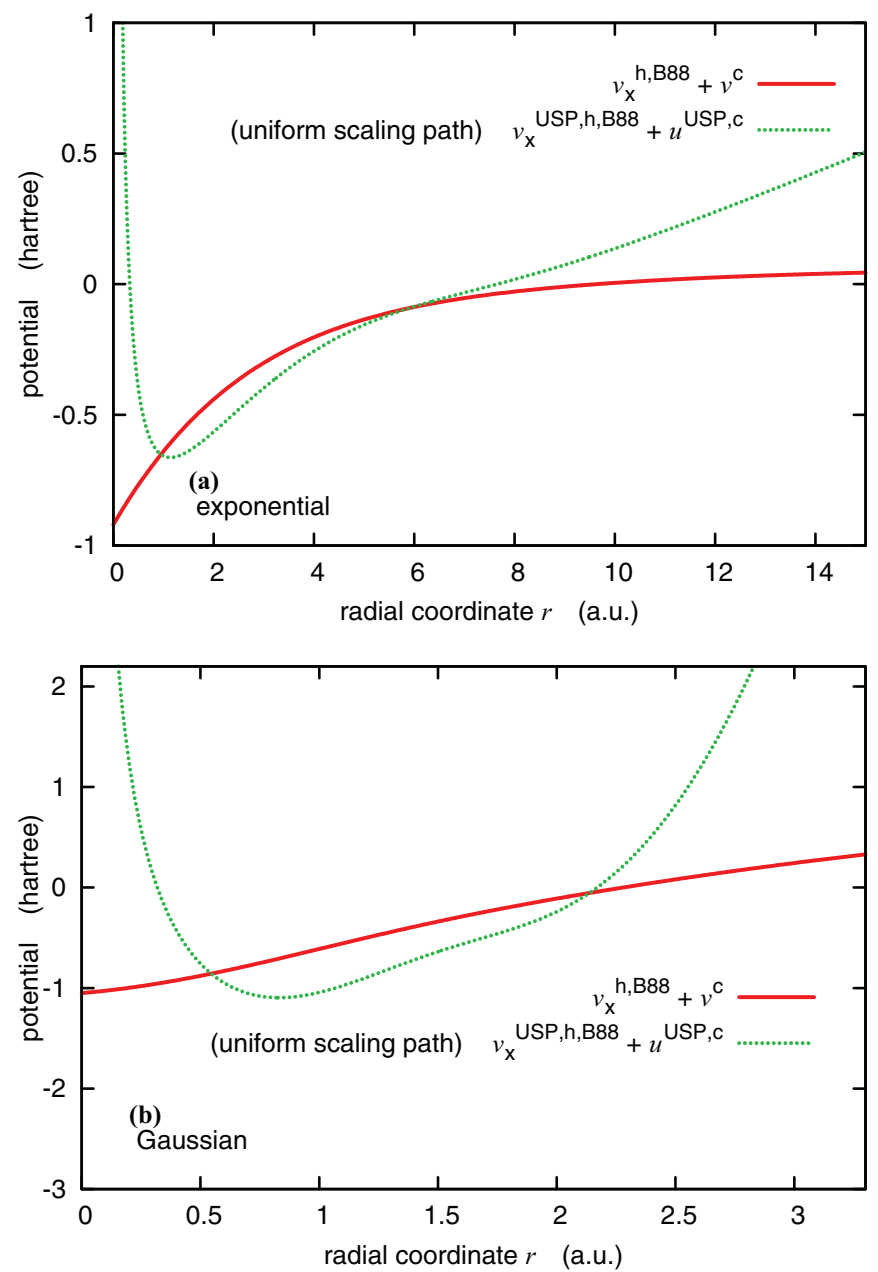

FIG. 7. (Color online) The potential expressions $v_{\mathrm{x}}^{\mathrm{h}, \mathrm{B} 88}+v^{\mathrm{c}}$ [Eqs. (A4) and (2)] and $v_{\mathrm{x}}^{\mathrm{USP}, \mathrm{h}, \mathrm{B} 88}+u^{\mathrm{USP}, \mathrm{c}}$ [Eqs. (B2) and (19)] for the exponential (a) and the Gaussian (b) spherical one-orbital densities.

decays exponentially. The finding that the transformations of BJ-type potentials do not preserve the good properties of the original potentials in such regions, and thus ruin one of the most attractive features of the BJ approach, is in itself already such a serious drawback that there is no point in going through the considerably more complicated many-orbital test.

One may speculate that the line integral transformation approach may be useful if the potential to which it is applied is already very close to a functional derivative in the mathematical sense. One possible route to achieve this could lie in the construction of a modified potential that already fulfills as closely as possible the constraints that a functional derivative would fulfill, e.g., the zero-force theorem [Eq. (6)] or the stronger constraint [50]

$$
\frac{\delta v(\mathbf{r})}{\delta \rho\left(\mathbf{r}^{\prime}\right)}=\frac{\delta v\left(\mathbf{r}^{\prime}\right)}{\delta \rho(\mathbf{r})} .
$$

However, one would need to find a modification of the BJ potential such that these expressions are fulfilled closely and at the same time the physics of the original BJ potential (e.g., the shell-structure steps) are not changed (e.g., similar to the idea of Ref. [53]). As $v^{\mathrm{BJ}}$ is already close to the exact Kohn-Sham exchange potential in many ground-state situations, only those modifications would be helpful that "add an almost zero term" to the potential in these situations. Finding such modifications is not at all an easy task.

Therefore, one may resort to an alternative approach of exploiting the attractive features that are undoubtedly present in the BJ potential. By analyzing how the BJ potential achieves the derivative discontinuity and the shell-structure steps one may be able to build these features into a semilocal energy functional from which the potential is then obtained in the usual way of taking a functional derivative. Recent progress in developing a generalized gradient approximation functional that shows shell structure and exchange discontinuities [54] suggests that it is a worthwhile task to further explore this option. For addressing the CT problem, e.g., it appears as a promising direction of future work to extend the new generalized gradient approximation ideas of Ref. [54] along the lines of Ref. [17].

\section{ACKNOWLEDGMENTS}

S.K. and A.K. acknowledge financial support from German Science Foundation GRK 1640 and the German Israeli Foundation, and A.K. acknowledges discussions with J. P. Perdew during a visit to Tulane University. R.A. acknowledges support from the Swedish Research Council (VR), Grant No. 621-2011-4249 and the Linnaeus Environment at Linköping on Nanoscale Functional Materials (LiLi-NFM) funded by VR.

\section{APPENDIX A: EXCHANGE HOLE POTENTIAL APPROXIMATIONS}

In this Appendix we give an overview of the exchange hole potential approximations used in Sec. III. The potentials are shown in spin-polarized notation for consistency with earlier literature.

LDA exchange hole potential:

$$
v_{\mathrm{x} \sigma}^{\mathrm{h}, \mathrm{LDA}}(\mathbf{r})=-3\left(\frac{3}{4 \pi}\right)^{1 / 3} \rho_{\sigma}^{1 / 3} .
$$

BR exchange hole potential [44]:

$$
v_{\mathrm{x} \sigma}^{\mathrm{h}, \mathrm{BR}}(\mathbf{r})=-\frac{1-e^{-x}-\frac{1}{2} x e^{-x}}{b},
$$

where $b^{3}=\frac{x^{3} e^{-x}}{8 \pi \rho_{\sigma}}$ and $x$ is determined numerically from

$$
\frac{x e^{-2 x / 3}}{x-2}=\frac{2}{3} \pi^{2 / 3} \frac{\rho_{\sigma}^{5 / 3}}{Q_{\sigma}},
$$

where $Q_{\sigma}=\frac{1}{6}\left[\nabla^{2} \rho_{\sigma}-\gamma\left(4 \tau_{\sigma}-\frac{1}{2} \frac{\left(\nabla \rho_{\sigma}\right)^{2}}{\rho_{\sigma}}\right)\right]$ with $\gamma=0.8$ and $\tau_{\sigma}=\sum_{i=1}^{N} \frac{1}{2}\left|\nabla \varphi_{i \sigma}\right|^{2}$.

B88 exchange hole potential [45]:

$$
v_{\mathrm{x} \sigma}^{\mathrm{h}, \mathrm{B} 88}(\mathbf{r})=v_{\mathrm{x} \sigma}^{\mathrm{h}, \mathrm{LDA}}(\mathbf{r})-2 \beta \rho_{\sigma}^{1 / 3} \frac{x_{\sigma}^{2}}{1+6 \beta x_{\sigma} \sinh ^{-1}\left(x_{\sigma}\right)}
$$

with $x_{\sigma}=\frac{\left|\nabla \rho_{\sigma}\right|}{\rho_{\sigma}^{4 / 3}}$ and $\beta=0.0042$. 
PBE exchange hole potential [46]:

$$
\begin{aligned}
& v_{\mathrm{x}}^{\mathrm{h}, \mathrm{PBE}}(\mathbf{r},[\rho])=2 A_{\mathrm{x}} \rho^{1 / 3}\left[1+\kappa-\frac{\kappa}{1+\frac{\mu s^{2}}{\kappa}}\right], \\
& v_{\mathrm{x} \sigma}^{\mathrm{h}, \mathrm{PBE}}\left(\mathbf{r},\left[\rho_{\sigma}\right]\right)=v_{\mathrm{x}}^{\mathrm{h}, \mathrm{PBE}}\left(\mathbf{r},\left[2 \rho_{\sigma}\right]\right)
\end{aligned}
$$

with $s=\frac{|\nabla \rho|}{2\left(3 \pi^{2}\right)^{1 / 3} \rho^{4 / 3}}, A_{\mathrm{x}}=-\frac{3}{4}\left(\frac{3}{\pi}\right)^{1 / 3}, \kappa=0.804$, and $\mu=$ 0.21951 .

PW86 exchange hole potential [47]:

$$
\begin{aligned}
& v_{\mathrm{x}}^{\mathrm{h}, \mathrm{PW} 86}(\mathbf{r},[\rho])=2 A_{\mathrm{x}} \rho^{1 / 3}\left[1+0.0864 \frac{s^{2}}{m}+b s^{4}+c s^{6}\right]^{m}, \\
& v_{\mathrm{x} \sigma}^{\mathrm{h}, \mathrm{PW} 86}\left(\mathbf{r},\left[\rho_{\sigma}\right]\right)=v_{\mathrm{x}}^{\mathrm{h}, \mathrm{PW} 86}\left(\mathbf{r},\left[2 \rho_{\sigma}\right]\right)
\end{aligned}
$$

with $m=\frac{1}{15}, b=14$, and $c=0.2$. For definitions of $s$ and $A_{\mathrm{x}}$ see above.

B86 exhange hole potential [48]:

$$
v_{\mathrm{x} \sigma}^{\mathrm{h}, \mathrm{B} 86}(\mathbf{r})=v_{\mathrm{x} \sigma}^{\mathrm{h}, \mathrm{LDA}}(\mathbf{r})-2 \beta \rho_{\sigma}^{1 / 3} \frac{x_{\sigma}^{2}}{1+\gamma x_{\sigma}^{2}}
$$

with $x_{\sigma}=\frac{\left|\nabla \rho_{\sigma}\right|}{\rho_{\sigma}^{4 / 3}}$ and $\beta=0.0036$ and $\gamma=0.004$.

\section{APPENDIX B: TRANSFORMATION OF THE B88 EXCHANGE HOLE POTENTIAL}

In this Appendix we transform the B88 exchange hole potential of Eq. (A4) with the transformation defined by Eqs. (10) and (11) for the uniform scaling path [Eq. (13)]. Since the B88 hole potential fulfills the exchange scaling relation [Eq. (17)] we can write for the energy

$$
E^{\mathrm{USP}, \mathrm{h}, \mathrm{B} 88}=\sum_{\sigma} \int d^{3} r v_{\mathrm{x} \sigma}^{\mathrm{h}, \mathrm{B} 88}(\mathbf{r})\left[3 \rho_{\sigma}(\mathbf{r})+\mathbf{r} \cdot \nabla \rho_{\sigma}(\mathbf{r})\right] .
$$

From this energy we obtain the functional derivative

$$
\begin{aligned}
v_{\mathrm{x} \sigma}^{\mathrm{USP}, \mathrm{h}, \mathrm{B} 88}= & \frac{\delta E^{\mathrm{USP}, \mathrm{h}, \mathrm{B} 88}}{\delta \rho_{\sigma}(\mathbf{r})} \\
= & \left(\frac{1}{3} \rho_{\sigma}^{-2 / 3} M_{\sigma}+3 \rho_{\sigma}^{1 / 3}\right)\left(C-2 \beta Q_{\sigma}\right) \\
& +2 \beta \nabla\left[\frac{\nabla \rho_{\sigma}}{\rho_{\sigma}\left|\nabla \rho_{\sigma}\right|} M_{\sigma} \frac{d Q_{\sigma}}{d x_{\sigma}}\right] \\
& +\frac{8}{3} \beta \frac{\left|\nabla \rho_{\sigma}\right|}{\rho_{\sigma}^{2}} M_{\sigma} \frac{d Q_{\sigma}}{d x_{\sigma}}-\nabla\left[\rho_{\sigma}^{1 / 3}\left(C-2 \beta Q_{\sigma}\right) \mathbf{r}\right],
\end{aligned}
$$

where

$$
\begin{gathered}
Q_{\sigma}=\frac{x_{\sigma}^{2}}{1+6 \beta x_{\sigma} \sinh ^{-1} x_{\sigma}} \\
\frac{d Q_{\sigma}}{d x_{\sigma}}=\frac{2 Q_{\sigma}}{x_{\sigma}}-\frac{6 \beta Q_{\sigma}^{2}}{x_{\sigma} \sqrt{1+x_{\sigma}^{2}}}-\frac{6 \beta Q_{\sigma}^{2} \sinh ^{-1}\left(x_{\sigma}\right)}{x_{\sigma}^{2}}, \\
M_{\sigma}=3 \rho_{\sigma}+(\mathbf{r} \cdot \nabla) \rho_{\sigma} \quad \text { and } \quad C=-3\left(\frac{3}{4 \pi}\right)^{1 / 3} .
\end{gathered}
$$

[1] P. Hohenberg and W. Kohn, Phys. Rev. 136, B864 (1964).

[2] W. Kohn and L. J. Sham, Phys. Rev. 140, A1133 (1965).

[3] E. Runge and E. K. U. Gross, Phys. Rev. Lett. 52, 997 (1984).

[4] D. J. Tozer, J. Chem. Phys. 119, 12697 (2003).

[5] N. Maitra, J. Chem. Phys. 122, 234104 (2005).

[6] M. J. G. Peach, P. Benfield, T. Helgaker, and D. J. Tozer, J. Chem. Phys. 128, 044118 (2008).

[7] M. Hellgren and E. K. U. Gross, Phys. Rev. A 85, 022514 (2012).

[8] L. Kronik, T. Stein, S. Refaely-Abramson, and R. Baer, J. Chem. Theory Comput. 8, 1515 (2012).

[9] A. Karolewski, T. Stein, R. Baer, and S. Kümmel, J. Chem. Phys. 134, 151101 (2011).

[10] D. Hofmann, T. Körzdörfer, and S. Kümmel, Phys. Rev. Lett. 108, 146401 (2012).

[11] S. J. A. van Gisbergen, P. R. T. Schipper, O. V. Gritsenko, E. J. Baerends, J. G. Snijders, B. Champagne, and B. Kirtman, Phys. Rev. Lett. 83, 694 (1999).

[12] S. Kümmel, L. Kronik, and J. P. Perdew, Phys. Rev. Lett. 93, 213002 (2004).

[13] R. van Leeuwen, O. Gritsenko, and E. J. Baerends, Z. Phys. D 33, 229 (1995).

[14] D. Hofmann and S. Kümmel, Phys. Rev. B 86, 201109(R) (2012).

[15] J. P. Perdew, R. G. Parr, M. Levy, and J. L. Balduz, Jr., Phys. Rev. Lett. 49, 1691 (1982).
[16] A. D. Becke and E. R. Johnson, J. Chem. Phys. 124, 221101 (2006).

[17] R. Armiento, S. Kümmel, and T. Körzdörfer, Phys. Rev. B 77, 165106 (2008).

[18] A. Karolewski, R. Armiento, and S. Kümmel, J. Chem. Theory Comput. 5, 712 (2009).

[19] A. P. Gaiduk and V. N. Staroverov, J. Chem. Phys. 128, 204101 (2008).

[20] E. Räsänen, S. Pittalis, and C. R. Proetto, J. Chem. Phys. 132, 044112 (2010).

[21] F. Tran, P. Blaha, and K. Schwarz, J. Phys.: Condens. Matter 19, 196208 (2007).

[22] F. Tran and P. Blaha, Phys. Rev. Lett. 102, 226401 (2009).

[23] M. Mundt and S. Kümmel, Phys. Rev. Lett. 95, 203004 (2005).

[24] A. P. Gaiduk and V. N. Staroverov, J. Chem. Phys. 131, 044107 (2009).

[25] J. B. Krieger, Y. Li, and G. J. Iafrate, Phys. Rev. A 45, 101 (1992).

[26] R. T. Sharp and G. K. Horton, Phys. Rev. 90, 317 (1953).

[27] J. D. Talman and W. F. Shadwick, Phys. Rev. A 14, 36 (1976).

[28] S. Kümmel and L. Kronik, Rev. Mod. Phys. 80, 3 (2008).

[29] H. Ou-Yang and M. Levy, Phys. Rev. Lett. 65, 1036 (1990).

[30] M. Levy and J. P. Perdew, Phys. Rev. A 32, 2010 (1985).

[31] M. Mundt, S. Kümmel, R. van Leeuwen, and P.-G. Reinhard, Phys. Rev. A 75, 050501 (2007).

[32] M. Mundt and S. Kümmel, Phys. Rev. A 74, 022511 (2006).

[33] M. Mundt and S. Kümmel, Phys. Rev. B 76, 035413 (2007). 
[34] L. Kronik, A. Makmal, M. L. Tiago, M. M. G. Alemany, M. Jain, X. Huang, Y. Saad, and J. R. Chelikowsky, Phys. Status Solidi B 243, 1063 (2006).

[35] K. Yabana and G. F. Bertsch, Phys. Rev. B 54, 4484 (1996).

[36] M. Lein and S. Kümmel, Phys. Rev. Lett. 94, 143003 (2005).

[37] N. T. Maitra, K. Burke, and C. Woodward, Phys. Rev. Lett. 89, 023002 (2002).

[38] F. Calvayrac, P.-G. Reinhard, and E. Suraud, Ann. Phys. 255, 125 (1997).

[39] P. M. Dinh, J. Messud, P.-G. Reinhard, and E. Suraud, J. Phys.: Conf. Ser. 248, 012024 (2010).

[40] D. Hofmann and S. Kümmel, J. Chem. Phys. 137, 064117 (2012).

[41] W. R. Fredrickson and W. W. Watson, Phys. Rev. 30, 429 (1927).

[42] S. P. Sinha, Proc. Phys. Soc. A 62, 124 (1949).

[43] J. C. Slater, Phys. Rev. 81, 385 (1951).
[44] A. D. Becke and M. R. Roussel, Phys. Rev. A 39, 3761 (1989). [45] A. D. Becke, Phys. Rev. A 38, 3098 (1988).

[46] J. P. Perdew, K. Burke, and M. Ernzerhof, Phys. Rev. Lett. 77, 3865 (1996).

[47] J. P. Perdew and Y. Wang, Phys. Rev. B 33, 8800 (1986).

[48] A. D. Becke, J. Chem. Phys. 84, 4524 (1986).

[49] Contrary to the exchange hole potential the exchange potential of B88 decays asymptotically with $1 / r^{2}$.

[50] R. van Leeuwen and E. J. Baerends, Phys. Rev. A 51, 170 (1995).

[51] R. van Leeuwen and E. J. Baerends, Phys. Rev. A 49, 2421 (1994).

[52] A. P. Gaiduk and V. N. Staroverov, J. Chem. Phys. 136, 064116 (2012).

[53] Y. Kurzweil and M. Head-Gordon, Phys. Rev. A 80, 012509 (2009).

[54] R. Armiento and S. Kümmel, Phys. Rev. Lett. 111, 036402 (2013). 